\title{
Resveratrol alleviates oxygen/glucose deprivation/reoxygenation-induced neuronal damage through induction of mitophagy
}

\author{
MING YE ${ }^{1}$, HUI WU ${ }^{2}$ and SHUGUO LI ${ }^{1}$ \\ ${ }^{1}$ Department of Geriatrics, Yichang Central People's Hospital; ${ }^{2}$ The First College of Clinical Medical Science, \\ China Three Gorges University, Yichang, Hubei 443003, P.R. China
}

Received February 7, 2020; Accepted October 19, 2020

DOI: $10.3892 / \mathrm{mmr} .2020 .11711$

\begin{abstract}
Resveratrol confers neuroprotective effects in cerebral ischemia; however, the involvement of mitophagy in the neuroprotective function of resveratrol remains unclear. The aim of the present study was to investigate whether resveratrol exerts neuroprotective effects on primary cortical neurons subjected to oxygen/glucose deprivation/reoxygenation (OGD/R) via modulating mitophagy. The data demonstrated that resveratrol at 1-10 $\mu \mathrm{M}$ during reoxygenation improved cell viability and suppressed apoptosis following OGD/R in a concentration-dependent manner. Moreover, resveratrol alleviated OGD/R-induced loss of mitochondrial membrane potential and excessive oxidative stress. Confocal imaging of LC3 and TOM20 antibody-labeled mitochondria, as well as western blot analysis, demonstrated that mitophagy was further enhanced following resveratrol treatment. In addition, resveratrol was revealed to stimulate the phosphatase and tensin homolog-induced kinase 1/Parkin pathway. Mitophagy inhibition then inhibited the protective effects of resveratrol. These results indicated that resveratrol exerts its protective effects against OGD/R damage, at least in part, by promoting mitophagy.
\end{abstract}

\section{Introduction}

Cerebral ischemia is associated with a number of serious diseases, including stroke and cardiac and respiratory arrest. Treatment usually involves blood flow recovery as soon as possible; however, this may lead to secondary injury in the ischemic region, referred to as ischemia/reperfusion injury (IRI) (1-3). Following cerebral ischemia, the recovery of the

Correspondence to: Dr Ming Ye, Department of Geriatrics, Yichang Central People's Hospital, 183 Yiling Road, Yichang, Hubei 443003, P.R. China

E-mail: geriatric_yeming@163.com

Key words: resveratrol, oxygen/glucose deprivation/reoxygenation, mitophagy, phosphatase and tensin homolog-induced kinase 1 blood circulation leads to inflammation and oxidative stress damage in areas affected by hypoxia and nutrient deficiency. Cerebral ischemia and reperfusion also lead to impaired mitochondrial oxidative metabolism and energy depletion in neurons, leading to programmed cell death $(4,5)$. Therefore, although cerebral ischemia treatment involves the restoration of blood flow as quickly as possible, this may induce additional injury to the ischemic area. Appropriate drugs are required to protect neurons from the effects of IRI, mitigate pathological responses and control the process of neuronal death (6). However, only few drugs to date are clinically available for patients with cerebral ischemia (6).

Resveratrol is a natural phytoalexin extracted from plants that exhibits neuroprotective, anticancer and anti-inflammatory properties (7-9). Resveratrol has also been reported to exhibit antioxidant properties, such as the ability to regulate nitric oxide metabolism (10) and chemopreventive activity (11). Moreover, resveratrol has been demonstrated to protect against IRI in gerbils (12). The beneficial neuroprotective effects of resveratrol may be due to its antiplatelet aggregation and vasodilation and/or antioxidant activity (13). A previous study demonstrated that resveratrol improves mitochondrial function and protects against metabolic disease by activating sirtuin 1 and PPARG coactivator-1 $\alpha$ (14). However, whether resveratrol serves as a mitochondrial phagocytosis regulator in brain ischemia remains unclear.

Autophagy is a cellular housekeeping function, which is responsible for the degradation of a number of protein aggregates or damaged organelles and is involved in numerous pathophysiological processes, including ischemic disease (15). There is great interest in determining how autophagy selectively identifies and removes damaged mitochondria. For example, it has been reported that Parkin-dependent mitophagy is key for neuroprotection of ischemic preconditioning (16). Furthermore, deletion of the uncoupling protein 2 gene in endothelial cells may lead to excessive phosphatase and tensin homolog-induced kinase 1 (PINK1)-induced mitochondrial autophagy, resulting in insufficient mitochondrial biosynthesis and increased apoptosis of endothelial cells (17). Resveratrol is considered to be a key regulator of mitochondrial activity and has been reported to attenuate mitochondrial function impairment 
induced by oxidative stress via upregulating mitochondrial antioxidant enzymes and decreasing the production of reactive oxygen species (ROS) by organelles. Resveratrol also triggers mitochondrial biogenesis, ameliorating the mitochondria-associated bioenergetics status in mammalian cells (18). However, the association between the neuroprotective effects of resveratrol during $\mathrm{OGD} / \mathrm{R}$ and mitophagy remains elusive.

The aims of the present study were to describe the dose-dependent protective effects of resveratrol against OGD/R-induced neuronal death and mitochondrial dysfunction, elucidate the effect of resveratrol on mitophagic activity during $\mathrm{OGD} / \mathrm{R}$ and to investigate the involvement of the PINK1/Parkin pathway in the neuroprotective effects of resveratrol in $\mathrm{OGD} / \mathrm{R}$. The present results may provide novel insight into the neuroprotective mechanisms of resveratrol and identify the potential value of resveratrol in protecting the brain from I/R damage.

\section{Materials and methods}

Preparation of rat cortical cultures. The present study was approved by the Ethics Committee of the China Three Gorges University (Yichang, China). Cultures of rat cortical neurons were prepared as previously described (19). A total of 8 rat pups (male; weight, 5-8 g; age, 1-2 days; China Three Gorges University, Yichang, China) were housed in a vivarium that was maintained at a fixed temperature $\left(22-23^{\circ} \mathrm{C}\right)$ and moisture(70\%), with a 12-h light/dark cycle and free access to food and drinking water. All procedures adhered to the National Institutes of Health Guidelines for the care and use of laboratory animals (20). The rat pups were euthanized by carbon dioxide overexposure. Once pups stopped moving (including respiration), all pups were sterilized in $75 \%$ ethanol and decapitated using sharp scissors, then the brains of the neonatal rats were removed in a sterile field. The cerebral cortex and hippocampus were isolated on ice for the preparation of primary astrocytes and neurons, respectively. After removing the meninges and blood vessels, the cerebral cortex and hippocampus were cut into $1-\mathrm{mm}^{3}$ pieces and digested with $2 \mathrm{mg} / \mathrm{ml}$ papain and $0.05 \mathrm{mg} / \mathrm{ml}$ DNase in serum-free medium at $37^{\circ} \mathrm{C}$ for $30 \mathrm{~min}$. The tissue suspension was gently pipetted to disperse the cells and filtered through a $100-\mu \mathrm{M}$ sterile filter. Neocortical cells were plated in dishes at $37^{\circ} \mathrm{C}$ at a density of $4 \times 10^{5}$ cells $/ \mathrm{ml}$ in DMEM with $10 \%$ FBS, epidermal growth factor $(10 \mathrm{ng} / \mathrm{ml})$, penicillin $(50 \mathrm{U} / \mathrm{ml})$ and streptomycin (50 U/ml; all Sigma-Aldrich; Merck KGaA). The neurons were initially cultured for 2 days (passage 0 , day 2; P0D2) and subcultured for 4 days (passage 1, day 4; P1D4). Neurons at the second passage (P2D4) were used for further study.

Cell culture and treatment. Following serum starvation, neurons were transferred to hypoxic incubators at $37^{\circ} \mathrm{C}$ for $4 \mathrm{~h}$ containing $1 \% \mathrm{O}_{2}, 5 \% \mathrm{CO}_{2}$ and $94 \% \mathrm{~N}_{2}$ to simulate ischemic conditions. For reoxygenation, the hypoxic cells were cultured in fresh medium and transferred to a normoxic incubator at $37^{\circ} \mathrm{C}$ for $2 \mathrm{~h}$. Resveratrol in the presence or absence $10 \mu \mathrm{M}$ mitochondrial division inhibitor-1 (Mdivi-1) was administered immediately prior to reoxygenation.
RNA interference (RNAi) and transfection. Small interfering (si)RNAs against PINK1 (siPINK1; 5'GCUAGUUACAAG AGAACAA-3'), Parkin (siParkin; 5'-GAAUACAUUCCCUAC CUCA-3') and scrambled siRNA (si-Control; 5'-UUCUCC GAACGUGUCACGU-3') were designed and synthesized by Shanghai GenePharma Co., Ltd. A total of $1 \times 10^{6}$ cells were transfected with $50 \mathrm{pg} / \mu 1$ siRNA, miRNA mimic, miRNA inhibitor or corresponding NCs using Lipofectamine ${ }^{\circledR}$ RNAiMAX transfection reagent (Thermo Fisher Scientific, Inc.) and harvested 48-72 $\mathrm{h}$ after transfection.

Annexin V-phycoerythrin (PE) and 7-aminoactinomycin D (7-AAD) staining. The cells were washed twice with cold PBS, then resuspended with $1 \mathrm{X}$ binding buffer. Then, $100 \mu 1$ cell suspension was transferred to 5-ml culture tubes. Annexin V-PE and 7-AAD (5 $\mu 1$ each; BD Biosciences) were added to each tube. The cells were vortexed gently and incubated for $15 \mathrm{~min}$ at room temperature in dark. A total of $400 \mu \mathrm{l}$ $1 \mathrm{X}$ binding buffer (BD Biosciences) was added to each tube. The reaction was performed at room temperature and in the dark for 10 min. Flow cytometry (BD Biosciences) was used to measure cells and data were analyzed using a FACSCanto II flow cytometer (BD Biosciences).

Cell viability assay. Cell survival rate was determined by Cell Counting Kit-8 (CCK-8; Beyotime Institute of Biotechnology) assay. After being grown in 96-well microplates at a density of 10,000 cells/well, the cells were treated with hypoxia/reoxygenation (H/R) and/or resveratrol as aforementioned. CCK-8 solution (10 $\mu \mathrm{l} /$ well) was added to each well and incubated in $5 \% \mathrm{CO}_{2}$ at $37^{\circ} \mathrm{C}$ for $1 \mathrm{~h}$. The absorbance was measured at $450 \mathrm{~nm}$. Optical density values were recorded as a ratio to the control.

Western blot analysis. Cell extracts were scraped into lysis buffer containing $20 \mathrm{mmol} / 1$ Tris- $\mathrm{HCl}(\mathrm{pH} 7.4), 6 \mathrm{mM}$ urea and $200 \mathrm{mmol} / \mathrm{l}$ potassium chloride with a protease inhibitor cocktail $(3.6 \mathrm{mmol} / 1$ leupeptin, $2.1 \mathrm{mmol} / \mathrm{l}$ pepstatin A and $50 \mathrm{mmol} / 1$ phenylmethylsulfonylfluoride) followed by vigorous vortexing and cooling on ice for $15 \mathrm{~min}$, followed by $15 \mathrm{~min}$ centrifugation at $13,000 \mathrm{x} \mathrm{g}$ at $4^{\circ} \mathrm{C}$. The protein concentration was determined using the Bradford (Thermo Fisher Scientific, Inc.) method, according to the manufacturer's instructions. The homogenates/lysates were stored at $-80^{\circ} \mathrm{C}$. Each group (20 $\mu \mathrm{g} / \mathrm{lane}$, the volume/lane was kept the same using loading buffer) was separated by $10 \%$ SDS-PAGE and transferred to PVDF membranes and blocked in TBST $(50 \mathrm{mM}$ Tris- $\mathrm{HCl}$, $\mathrm{pH} 7.5,150 \mathrm{mM} \mathrm{NaCl}$ and $0.2 \%$ Tween-20) containing $5 \%$ non-fat milk for $1 \mathrm{~h}$ at $37^{\circ} \mathrm{C}$. The antibodies used were as follows: Anti-LC3I/II (1:1,000; cat. no. 3868; Cell Signaling Technology, Inc.), anti-Caspase-3 (1:1,000; cat. no. 9662; Cell Signaling Technology, Inc.), anti-translocase of outer mitochondrial membrane (TOMM)20 (1:1,000; cat. no. ab186734; Abcam), anti-translocase of inner mitochondrial membrane (TIMM)23 (1:1,000; cat. no. sc-13298; Santa Cruz Biotechnology, Inc.) and GAPDH (1:4,000; cat. no. GB11002; Wuhan Servicebio Technology Co., Ltd.) prior to incubation overnight at $4^{\circ} \mathrm{C}$. The blots were then incubated with the secondary antibody (1:5,000; cat. no. 7074; Cell Signaling Technology, Inc.) at room temperature for $1 \mathrm{~h}$. The experiments 
were repeated $\geq 3$ times and bands were detected using enhanced chemiluminescence (Bio-Rad Laboratories, Inc.). Band densities were semi-quantified using Gel-Pro Analyzer densitometry software (version 6.3; Media Cybernetics, Inc.).

Mitochondrial function assays. In order to investigate mitochondrial superoxide production and membrane potential $(\Delta \Psi \mathrm{m})$, the cells were incubated at $37^{\circ} \mathrm{C}$ with MitoSOX $(0.5 \mu \mathrm{M} \mathrm{mol} / \mathrm{l}$; Thermo Fisher Scientific, Inc.) or $50 \mathrm{nmol} / \mathrm{l}$ tetramethylrhodamine ethyl ester (TMRE; Thermo Fisher Scientific, Inc.) for $15 \mathrm{~min}$. Fluorescence images for TMRE and MitoSOX were obtained using confocal laser scanning microscope (magnification, x200; Leica TCS SP2; Leica Microsystems $\mathrm{GmbH}$ ). The average fluorescent intensity for each group was analyzed with ImageJ software (version 1.41, National Institutes of Health).

Immunofluorescence. Imaging studies for GFP-LC3 were performed as previously described (21). Neurons were infected with adenovirus encoding GFP-LC3 (multiplicity of infection $=20$ ) at $37^{\circ} \mathrm{C}$ for $48 \mathrm{~h}$. After 2 days, the samples were fixed with $4 \%$ paraformaldehyde at $4^{\circ} \mathrm{C}$ for $30 \mathrm{~min}$, permeated with $70 \%$ methanol/acetone at $4^{\circ} \mathrm{C}$ for $30 \mathrm{~min}$ and stained with anti-TOM20 antibody (1:200; cat. no. ab186734; Abcam) at $4^{\circ} \mathrm{C}$ overnight. GFP-positive cells were selected for observation under an inverted fluorescence microscope in order to count the number of GFP-LC3 punctas. One puncta was regarded as equal to one autophagosome. The number of GFP dots was determined by manual counting the fluorescent puncta. The samples were then observed under a fluorescence confocal microscope (magnification, x400; LSM510 META; Carl Zeiss AG). Mander's overlap coefficient was used to quantify the degree of colocalization using Image Pro-Plus software (version 4.5) (22).

Statistical analysis. Data are expressed as the mean \pm SEM of $\geq 3$ independent repeats. An unpaired two-tailed t-test was used for comparisons between two groups. ANOVA or repeated ANOVA followed by Bonferroni's post hoc test was used to perform multiple comparisons using GraphPad Prism ${ }^{\circledR}$ software (version 6.0; GraphPad Software, Inc.). $\mathrm{P}<0.05$ was considered to indicate a statistically significant difference.

\section{Results}

Resveratrol inhibits OGD/R-induced decrease in cell viability and apoptosis. In order to study the neuroprotective effect of resveratrol on OGD/R-injured cells, a cell viability assay was first performed. Resveratrol at 1-30 $\mu \mathrm{M}$ administered at the beginning of reoxygenation restored cell viability in a concentration-dependent manner, compared with the OGD/R group (Fig. 1A). Flow cytometry was used to quantify OGD/R-induced neuronal apoptosis. Resveratrol inhibited OGD/R-induced apoptosis (Fig. 1B). Similarly, western blot analysis revealed that resveratrol treatment significantly decreased OGD/R-induced cleavage of the proapoptotic protein caspase-3 (Fig. 1C). Based on these results, $10 \mu \mathrm{M}$ resveratrol was used for subsequent experiments, as it appears to confer a greater protection.
Resveratrol attenuates $O G D / R$-induced oxidative stress and preserves mitochondrial function. During OGD/R, the levels of mitochondrial ROS (i.e. superoxide) increased, whereas these changes were attenuated by resveratrol (Fig. 2A). Subsequently, the effect of resveratrol on $\Delta \Psi$ m during OGD/R, which is a key event during cell death, was examined via assessing the intensity of TMRE fluorescence. The data indicated that resveratrol significantly promoted OGD/R-induced decrease in $\Delta \Psi \mathrm{m}$ (Fig. 2B). In summary, these data indicated that resveratrol prevented mitochondrial dysfunction in neuronal cells following OGD/R.

Resveratrol activates $O G D / R$-induced mitophagy. The occurrence of mitophagy was detected during OGD/R. Immunoblot analysis demonstrated that OGD/R significantly increased the levels of the autophagy-activated biochemical marker LC3B-II. These changes in LC3B-II during OGD/R were associated with a significant decrease in levels of the mitochondrial inner membrane proteins TIMM23 and TOMM20 (Fig. 3A). Activation of mitochondrial autophagy, as well as decrease in mitochondrial mass, are implicated in the activation of mitochondrial autophagy (16). Resveratrol activated mitophagy, as indicated by changes in LC3B-II, TIMM23 and TOMM20 levels (Fig. 3A). In order to monitor mitochondrial autophagy in cortical cells, mitochondria were immunostained with anti-TOM20 antibody and autophagosomes were observed with GFP-LC3 punctate structures. Consistently, resveratrol increased the overlap of mitochondria and autophagosomes in OGD/R-injured cells compared with that in the control group (Fig. 3B). Collectively, these results indicated that resveratrol enhanced OGD/R-induced mitochondrial autophagy.

PINK1/Parkin-mediated mitophagy is involved in the protective effects of resveratrol. In order to determine the mechanism underlying resveratrol-induced mitophagy, the effects of the PINK1/Parkin pathway, which serves a key role in mitophagy, were investigated during $\mathrm{OGD} / \mathrm{R}$. The expression levels of Parkin and PINK1 were first assessed during OGD/R in the presence or absence of resveratrol. The results indicated that resveratrol further enhanced the OGD/R-induced increase in PINK1 and Parkin protein expression levels (Fig. 4A). Therefore, the focus was placed on PINK1/Parkin to elucidate the mitophagy pathway implicated in the neuroprotective effects of resveratrol. In order to determine the role of PINK1/Parkin, their expression was silenced with specific siRNAs. Cells with PINK1 or Parkin knockdown exhibited lower mitophagy compared with control siRNA-transfected cells (data not shown). These results revealed that PINK1 and Parkin siRNA itself did not affect cell viability and the apoptosis rate (Fig. 4B and C). Consistently, the downregulation of PINK1 or Parkin reversed the the improved cell survival and decreased apoptosis rate caused by resveratrol in the OGD/R group (Fig. 4D and E). Collectively, these results indicated that PINK1/Parkin-mediated mitophagy was responsible for the neuroprotective effects of resveratrol.

Mitophagy inhibition blocks the protective effects of resveratrol on $O G D / R$-injured cells. In order to elucidate the contribution of mitophagy to the protective effects of resveratrol 
A

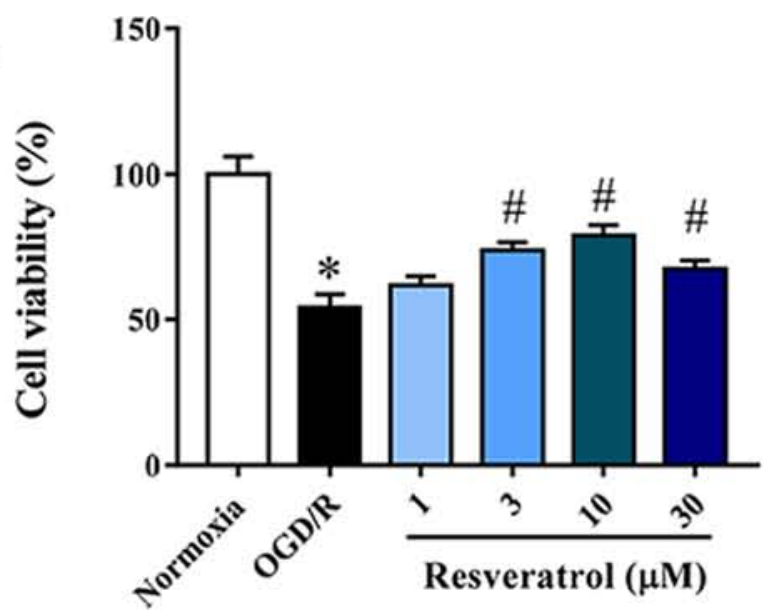

B
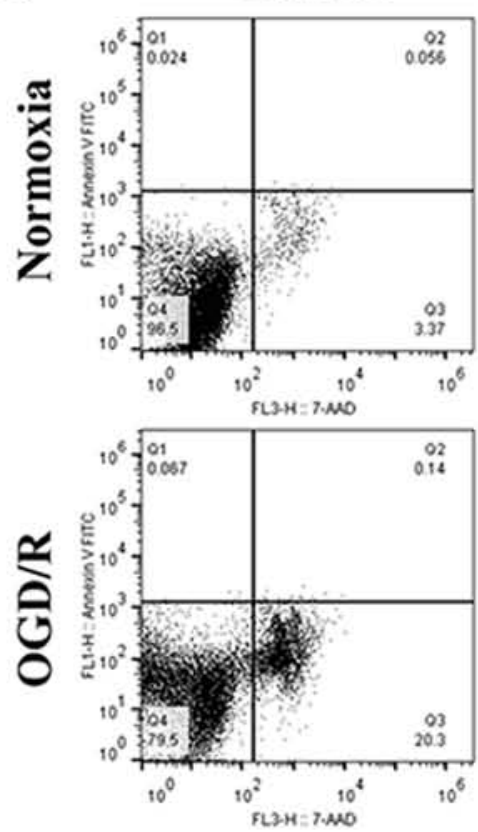

Control
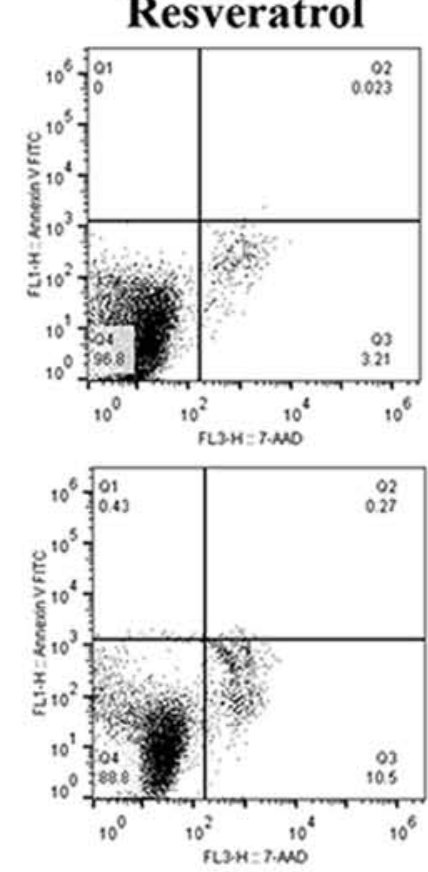

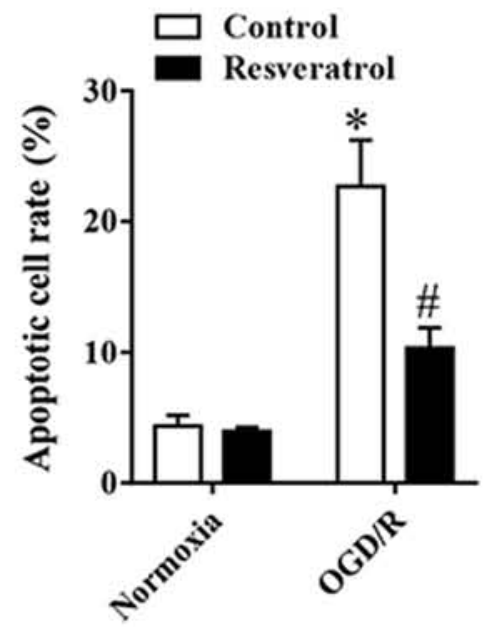

C
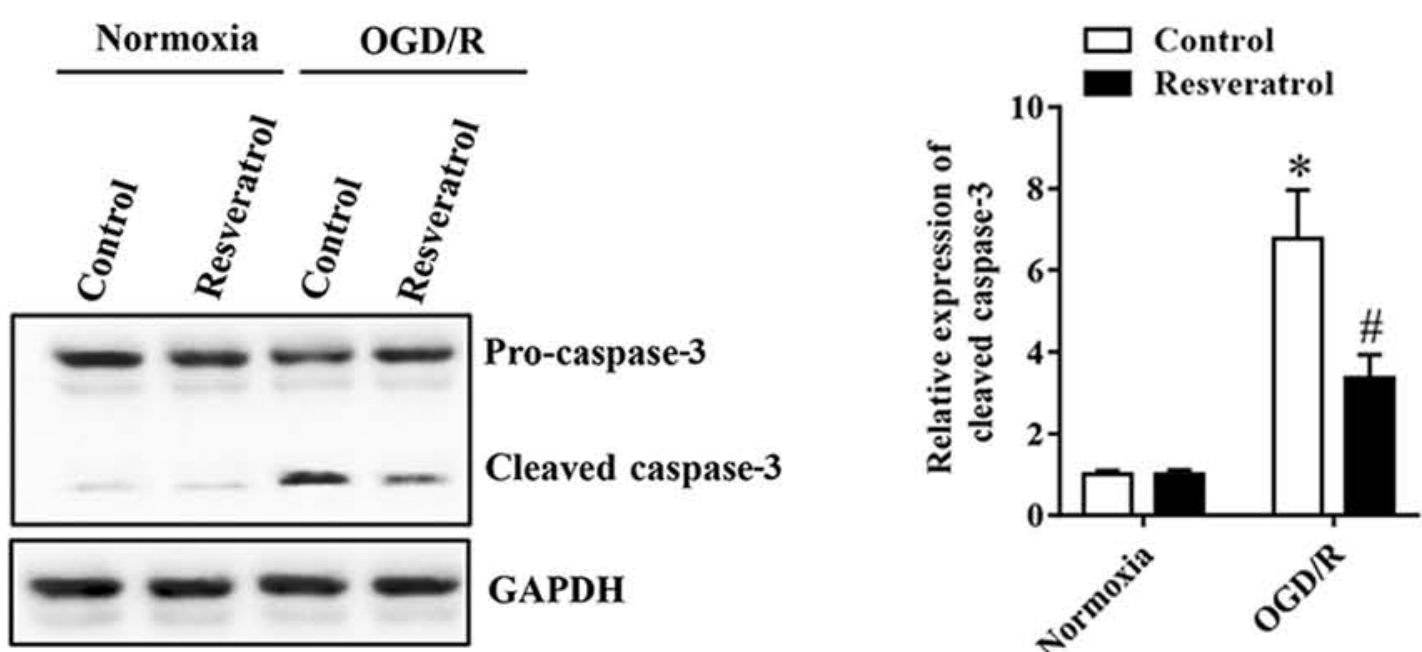

Figure 1. Resveratrol protects primary cortical neurons against OGD/R injury. (A) Resveratrol (1-10 $\mu \mathrm{M})$ increased cell viability in a concentration-dependent manner. Resveratrol inhibited OGD/R-induced cell apoptosis, as assessed by (B) flow cytometry and (C) western blotting. Data are presented as the mean \pm SEM $(n=3)$. ${ }^{*} \mathrm{P}<0.05$ vs. the control. ${ }^{~} \mathrm{P}<0.05$ vs. OGD/R. OGD/R, oxygen/glucose deprivation/reoxygenation. 
A

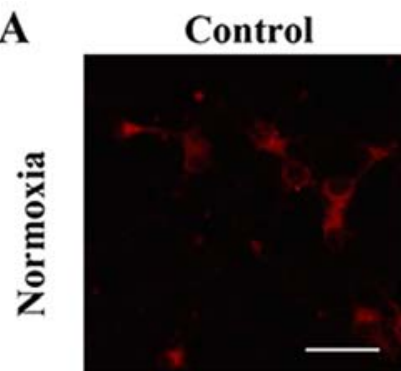

Resveratrol
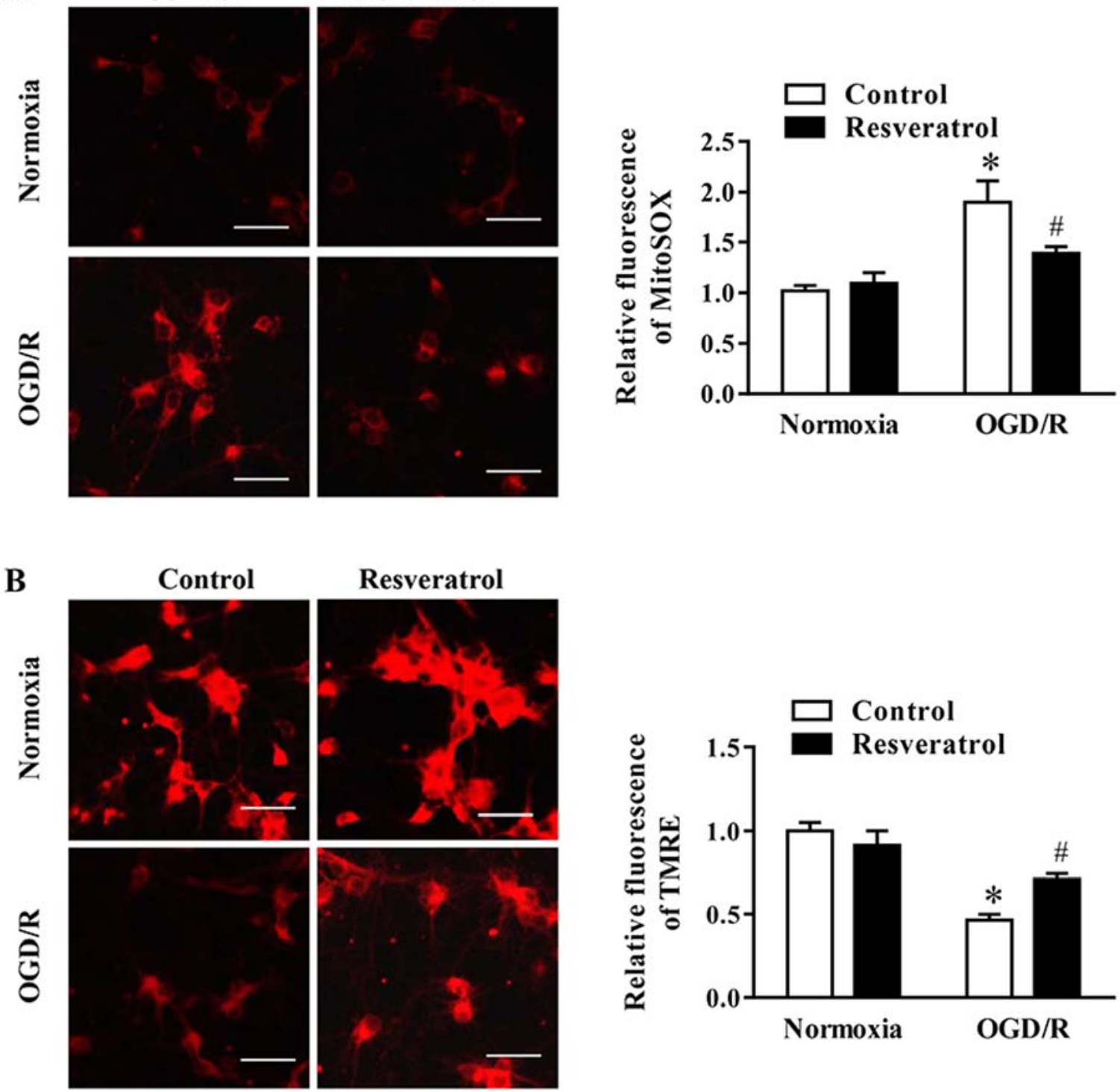

Figure 2. Effects of resveratrol on mitochondrial function and cell death following OGD/R. Representative images and quantification of fluorescence intensity of (A) mitochondrial superoxide and (B) TMRE in control and OGD/R-injured cells. Scale bar, $50 \mu \mathrm{m}$. Data are presented as the mean $\pm \mathrm{SEM}\left(\mathrm{n}=3\right.$ ). ${ }^{*} \mathrm{P}<0.05$ vs. the control. ${ }^{\text {P }}<0.05$ vs. OGD/R. OGD/R, oxygen/glucose deprivation/reoxygenation; TMRE, tetramethylrhodamine ethyl ester.

against OGD/R injury, the mitophagy inhibitor Mdivi-1 (23) was employed. Mdivi-1 reversed resveratrol-inhibited mitochondrial production of ROS during OGD/R (Fig. 5A). Concurrently, the resveratrol-protected $\Delta \Psi \mathrm{m}$ in simulated OGD/R cells was abolished with Mdivi-1 treatment (Fig. 5B). In addition, Mdivi-1 also reversed the resveratrol-induced inhibition of apoptosis and improvement in cell survival following OGD/R (Fig. 5C and D). In summary, these data demonstrated that enhanced mitophagy served a key role in the neuroprotective effects of resveratrol against OGD/R injury. Collectively, the present results provided evidence supporting the neuroprotective role of resveratrol during OGD/R, which was mediated, at least in part, via enhancing mitophagy.

\section{Discussion}

Stroke causes brain injury in millions of individuals worldwide each year (24). Although it is known that ischemia causes cellular damage, the underlying mechanisms are incompletely understood, and there is currently no approved therapy that decreases infarct size or neurological disability $(25,26)$. Pharmacological interference is more clinically feasible compared with a mechanical approach, as it is easier to implement and is less invasive (21). However, the availability of effective drugs for the treatment of cerebral IRI is currently limited. To the best of our knowledge, the present study is the first to demonstrate that resveratrol can protect neurons against $\mathrm{OGD} / \mathrm{R}$ via a mechanism that involves the modulation of mitochondrial autophagy. The primary findings were as follows: i) Resveratrol concentration-dependently protected neurons against OGD/R injury; ii) resveratrol protected primary neurons from decreased $\Delta \Psi \mathrm{m}$ and improved cell survival following OGD/R; iii) resveratrol exerted protective effects by promoting PINK1/Parkin-mediated mitophagy. These findings demonstrated the role and mechanisms of action of resveratrol in neuroprotection against OGD/R injury. 

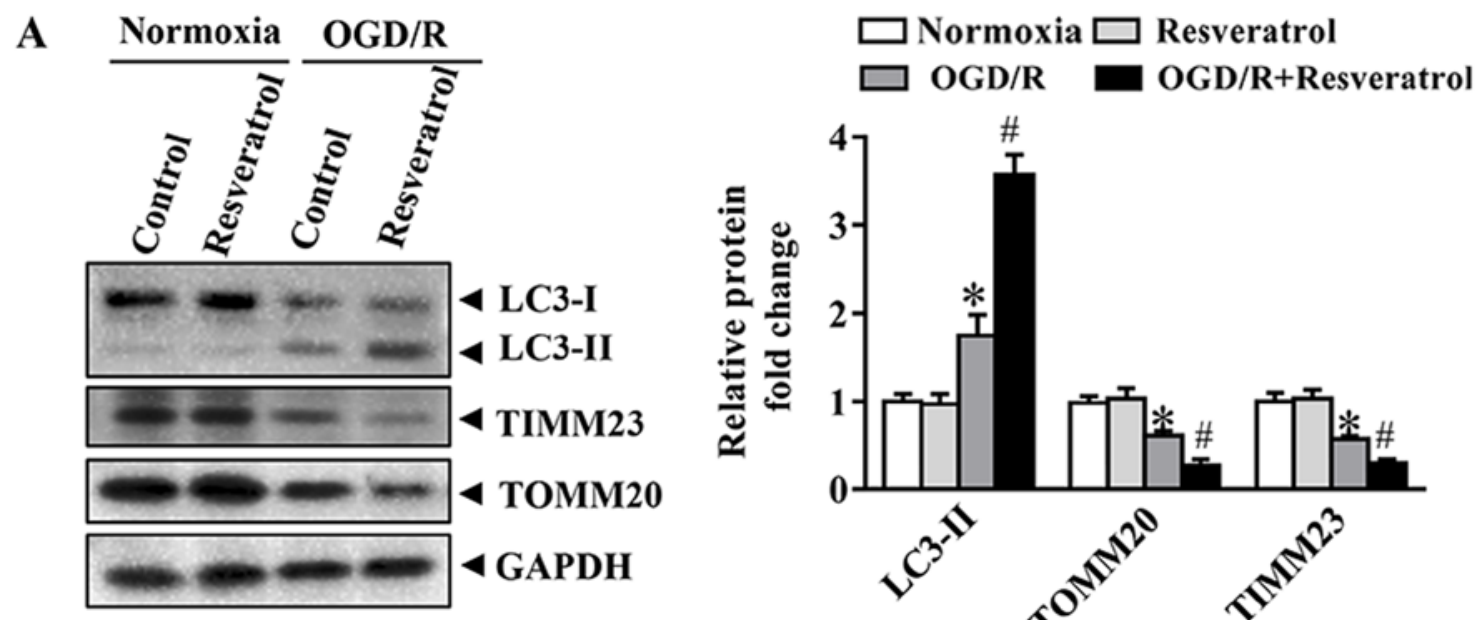

B
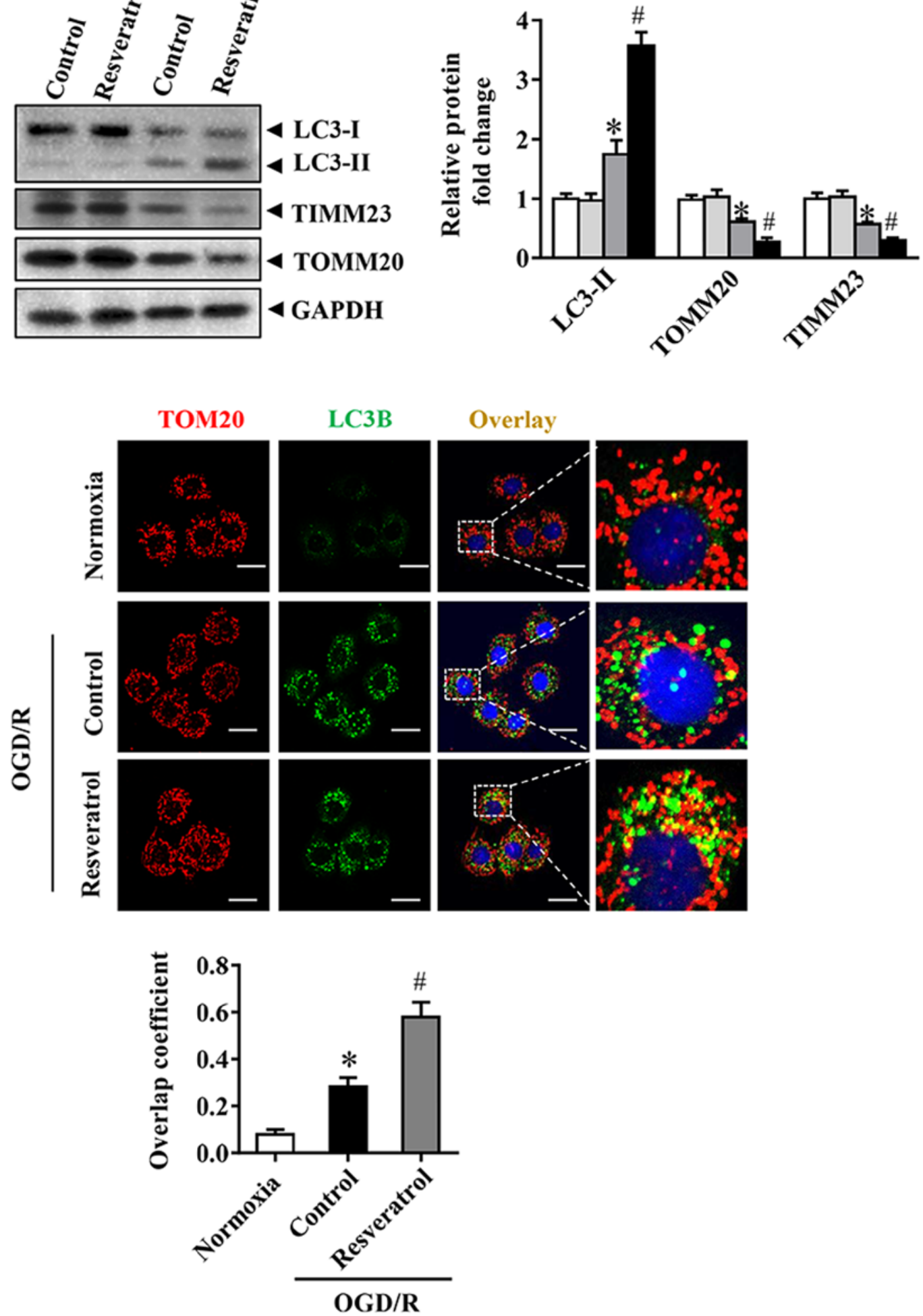

Figure 3. Effects of resveratrol on mitophagy induction following OGD/R in primary cortical neurons. (A) Representative immunoblots and data for LC3 and mitochondrial markers, including TOMM20 and TIMM23, as determined by western blotting. GAPDH was used as the loading control. (B) Representative immunostaining of cells for LC3B (green) and mitochondria with anti-TOM20 (red). Scale bar, $10 \mu \mathrm{m}$. Data are presented as the mean $\pm \mathrm{SEM}$ (n=3). ${ }^{*} \mathrm{P}<0.05$ vs. the control. " $\mathrm{P}<0.05$ vs. OGD/R. OGD/R, oxygen/glucose deprivation/reoxygenation; TOMM, translocase of outer mitochondrial membrane; TIMM, translocase of inner mitochondrial membrane.

Resveratrol is found in red wine and numerous edible plants, including grapes, peanuts and plums (10). Resveratrol has been demonstrated to have a number of biological functions, such as antiaging, anti-inflammatory, antioxidant, anticancer and antiapoptotic properties (27). The resveratrol-rich Mediterranean diet significantly decreases the risk 
A
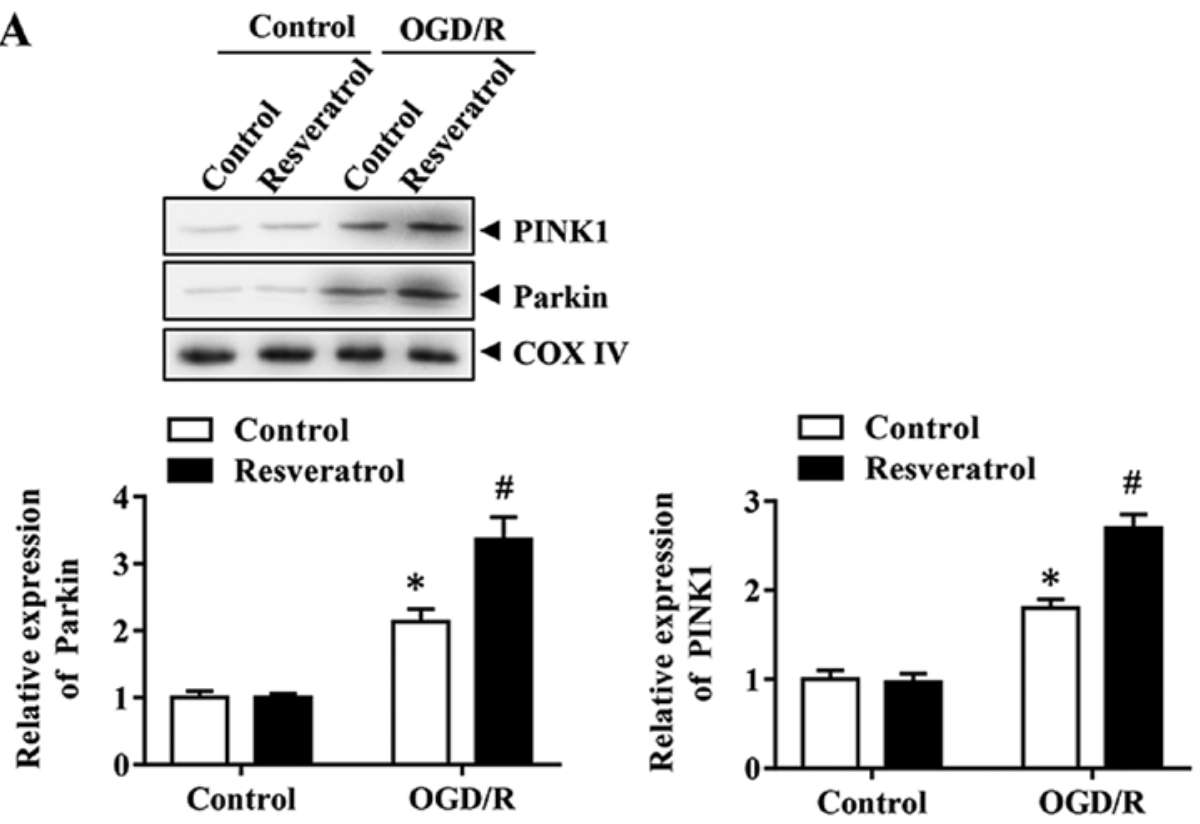

B
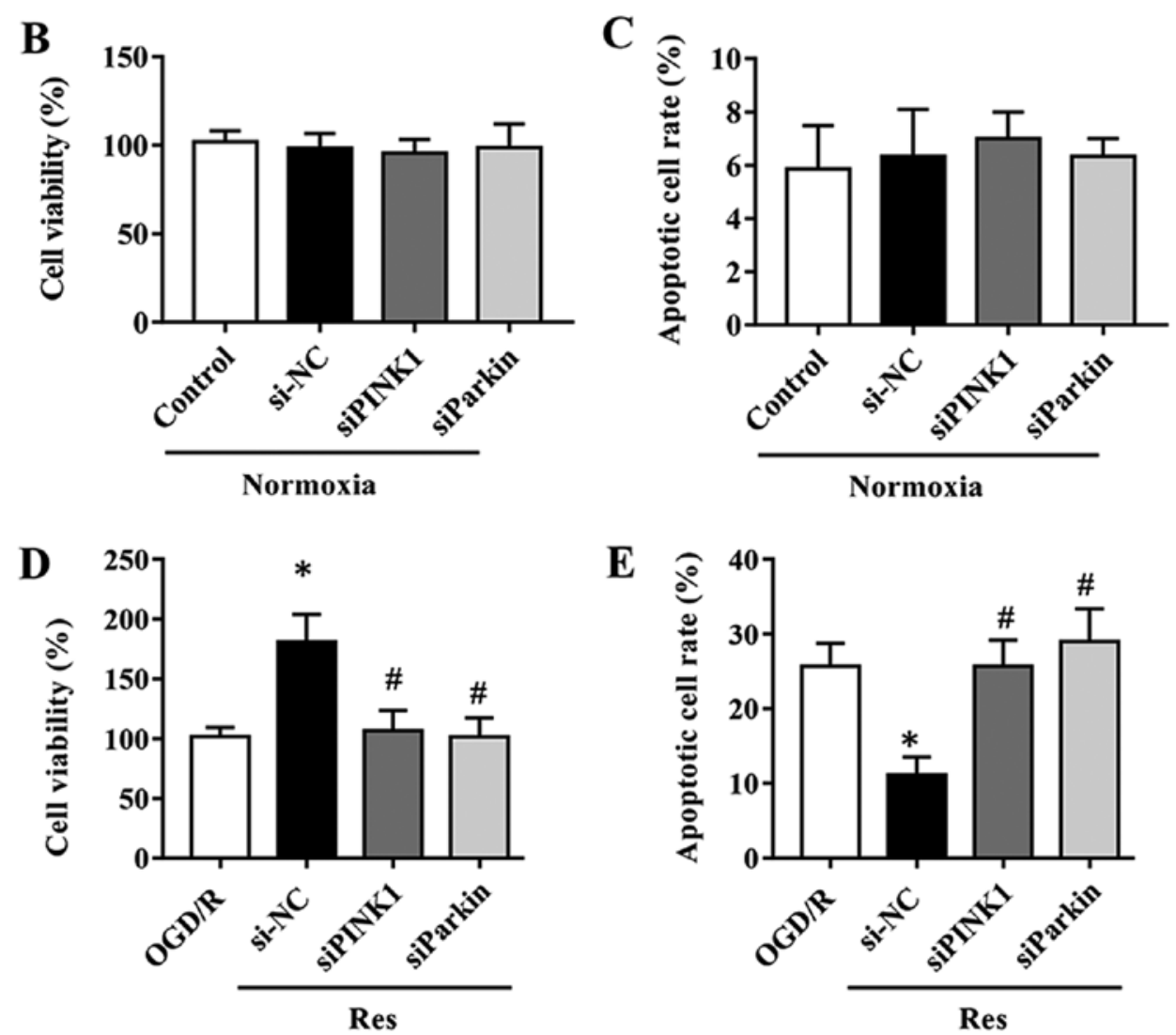

Figure 4. Effect of resveratrol on the PINK1/Parkin pathway in primary cortical neurons. (A) Expression levels of PINK1 and Parkin in OGD/R in the presence or absence of resveratrol were detected by immunoblotting. Effects of PINK1 or Parkin knockdown on the (B) viability and (C) apoptosis of control cells. Effects of PINK1 and Parkin silencing on (D) viability and (E) apoptosis of neurons subjected to OGD/R simulation. Data are presented as the mean \pm SEM $(\mathrm{n}=3)$. ${ }^{*} \mathrm{P}<0.05$ vs. the control. ${ }^{*} \mathrm{P}<0.05$ vs. OGD/R. OGD/R, oxygen/glucose deprivation/reoxygenation; PINK1, phosphatase and tensin homolog-induced kinase 1 ; si, small interfering; $\mathrm{NC}$, negative control.

of cardiovascular disease (28). Resveratrol pretreatment before ischemic brain injury decreases the loss of neurons and infarct size (29). In addition, resveratrol administration at $6 \mathrm{~h}$ after ischemia or reperfusion can also effectively decrease infarct size (30). These results provide a basis for the clinical application of resveratrol in the prevention and treatment of acute ischemic stroke. However, the exact underlying molecular mechanism is unclear. The present research indicated that resveratrol protected mitochondrial function and improved the cell survival rate when administered at the 


\section{A}
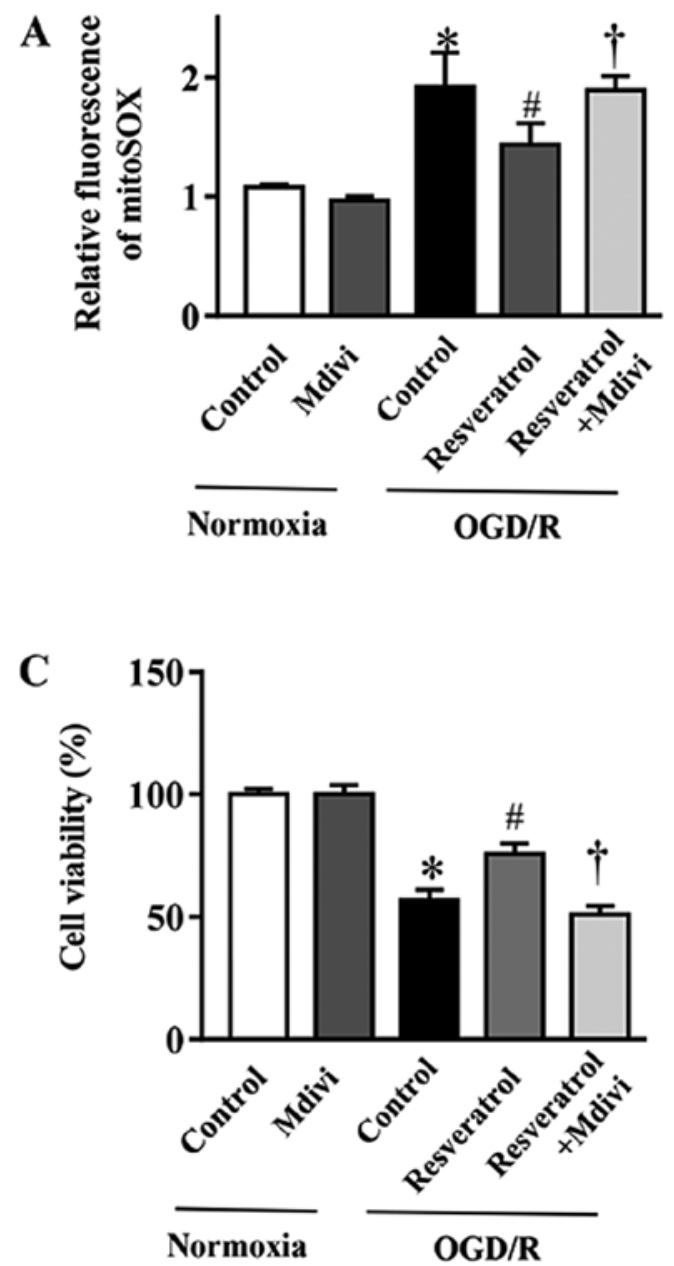

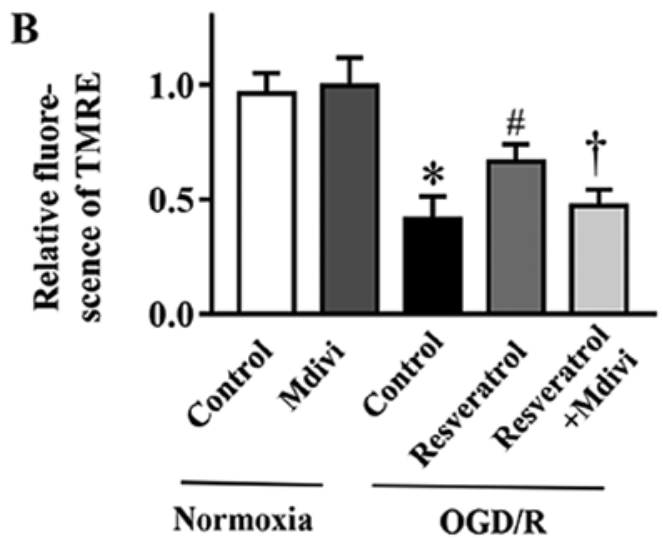

D

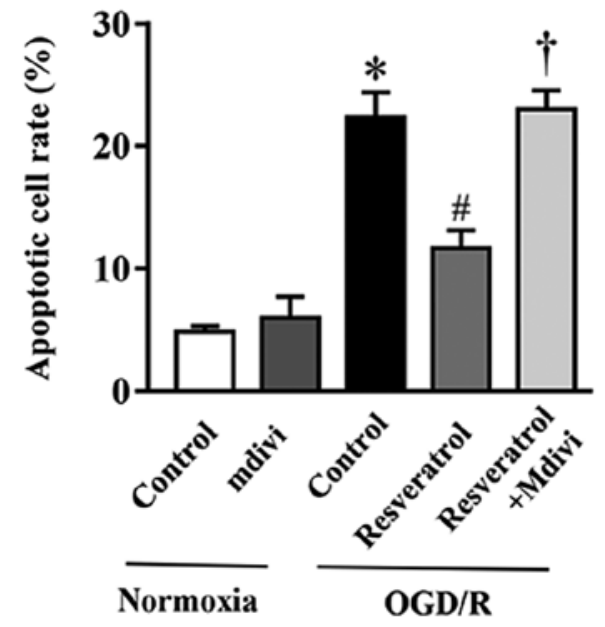

Figure 5. Effect of the mitophagy inhibitor Mdivi-1 on cell death and mitochondrial function in OGD/R-injured primary cortical neurons. (A) Quantification of fluorescence intensity of mitochondrial superoxide. (B) TMRE fluorescence intensity indicated mitochondrial membrane potential. (C) Viability and (D) apoptosis of neurons subjected to OGD/R simulation in the presence or absence of Mdivi-1. Data are presented as the mean $\pm \mathrm{SEM}\left(\mathrm{n}=3\right.$ ). ${ }^{*} \mathrm{P}<0.05$ vs. the control. ${ }^{\#} \mathrm{P}<0.05$ vs. OGD/R. ${ }^{\dagger} \mathrm{P}<0.05$ vs. OGD/R + resveratrol. OGD/R, oxygen/glucose deprivation/reoxygenation; Mdivi-1, mitochondrial division inhibitor-1; TMRE, tetramethylrhodamine ethyl ester.

beginning of reoxygenation, but the underlying mechanism remains elusive.

The balance between mitochondrial autophagy and biogenesis is a determinant of cell function disorder and disease $(31,32)$. The role of mitophagy in organ ischemic disease has been controversial (33-36). Mitochondrial autophagy is a selective form of autophagy that removes unwanted or damaged mitochondria $(37,38)$. PINK1/Parkin-mediated induction of mitophagy and its protective effects have been demonstrated in cerebral ischemia (39-41). However, little is known regarding the role of mitophagy in resveratrol-induced neuroprotection. The findings of the present study may provide novel insights into the underlying mechanisms of resveratrol and potential prevention strategies. It was observed that resveratrol activated PINK1/Parkin-mediated mitochondrial phagocytosis in primary cortical neurons, but further studies are required to elucidate the underlying mechanisms.

In summary, the present study demonstrated that resveratrol may be administered at the onset of reoxygenation and exerts neuroprotective effects that are partly mediated by activating the PINK1/Parkin signaling pathway in primary cortical neurons. Further research is required to elucidate the exact mechanism of neuroprotection of resveratrol and to determine the potential role of resveratrol in the treatment of cerebral ischemia.

\section{Acknowledgements}

Not applicable.

\section{Funding}

The present study was supported by a grant from the Medical and Health Research Project of Yichang (grant no. A17-301-13).

\section{Availability of data and materials}

The datasets used and/or analyzed during the current study are available from the corresponding author on reasonable request.

\section{Authors' contributions}

YM and WH contributed to study design, data collection, statistical analysis, data interpretation and manuscript preparation. 
WH, YM and SL contributed to data collection and statistical analysis. YM contributed to data collection, statistical analysis and manuscript preparation. All authors read and approved the final manuscript.

\section{Ethics approval and consent to participate}

The present study was approved by the Ethics Committee of the China Three Gorges University (approval no. 20180105181532).

\section{Patient consent for publication}

Not applicable.

\section{Competing interests}

The authors declare that they have no competing interests.

\section{References}

1. Puyal J, Ginet V and Clarke PG: Multiple interacting cell death mechanisms in the mediation of excitotoxicity and ischemic brain damage: A challenge for neuroprotection. Prog Neurobiol 105: 24-48, 2013.

2. Chen H, Yoshioka H, Kim GS, Jung JE, Okami N, Sakata H, Maier CM, Narasimhan P, Goeders CE and Chan PH: Oxidative stress in ischemic brain damage: Mechanisms of cell death and potential molecular targets for neuroprotection. Antioxid Redox Signal 14: 1505-1517, 2011.

3. Mehta SL, Manhas N and Raghubir R: Molecular targets in cerebral ischemia for developing novel therapeutics. Brain Res Rev 54: 34-66, 2007.

4. Cabral-Costa JV and Kowaltowski AJ: Neurological disorders and mitochondria. Mol Aspects Med 71: 100826, 2020.

5. Andrabi SS, Tabassum H, Parveen S and Parvez S: Ropinirole induces neuroprotection following reperfusion-promoted mitochondrial dysfunction after focal cerebral ischemia in Wistar rats. Neurotoxicology 77: 94-104, 2019.

6. Yang J, Wu S, Hou L, Zhu D, Yin S, Yang G and Wang Y: Therapeutic effects of simultaneous delivery of nerve growth factor mRNA and protein via exosomes on cerebral ischemia Mol Ther Nucleic Acids 21: 512-522, 2020.

7. Celotti E, Ferrarini R, Zironi R and Conte LS: Resveratrol content of some wines obtained from dried Valpolicella grapes: Recioto and Amarone. J Chromatogr A 730: 47-52, 1996.

8. Pany S, Majhi A and Das J: PKC activation by resveratrol derivatives with unsaturated aliphatic chain. PLoS One 7: e52888, 2012.

9. Das J, Pany S and Majhi A: Chemical modifications of resveratrol for improved protein kinase C alpha activity. Bioorg Med Chem 19: 5321-5333, 2011.

10. Carrizzo A, Puca A, Damato A, Marino M, Franco E, Pompeo F, Traficante A, Civitillo F, Santini L, Trimarco V and Vecchione C: Resveratrol improves vascular function in patients with hypertension and dyslipidemia by modulating NO metabolism. Hypertension 62: 359-366, 2013.

11. Kesherwani V, Atif F, Yousuf S and Agrawal SK: Resveratrol protects spinal cord dorsal column from hypoxic injury by activating Nrf-2. Neuroscience 241: 80-88, 2013.

12. Wang Q, Xu J, Rottinghaus GE, Simonyi A, Lubahn D, Sun GY and Sun AY: Resveratrol protects against global cerebral ischemic injury in gerbils. Brain Res 958: 439-447, 2002.

13. Gao D, Zhang X, Jiang X, Peng Y, Huang W, Cheng G and Song L: Resveratrol reduces the elevated level of MMP-9 induced by cerebral ischemia-reperfusion in mice. Life Sci 78: 2564-2570, 2006.

14. Lagouge M, Argmann C, Gerhart-Hines Z, Meziane H, Lerin C, Daussin F, Messadeq N, Milne J, Lambert P, Elliott P, et al: Resveratrol improves mitochondrial function and protects against metabolic disease by activating SIRT1 and PGC-1alpha. Cell 127: 1109-1122, 2006

15. Choi AM, Ryter SW and Levine B: Autophagy in human health and disease. N Engl J Med 368: 651-662, 2013.
16. Livingston MJ, Wang J, Zhou J, Wu G, Ganley IG, Hill JA, Yin XM and Dong Z: Clearance of damaged mitochondria via mitophagy is important to the protective effect of ischemic preconditioning in kidneys. Autophagy 15: 2142-2162, 2019.

17. Haslip M, Dostanic I, Huang Y,Zhang Y, Russell KS, Jurczak MJ, Mannam P, Giordano F, Erzurum SC and Lee PJ: Endothelial uncoupling protein 2 regulates mitophagy and pulmonary hypertension during intermittent hypoxia. Arterioscler Thromb Vasc Biol 35: 1166-1178, 2015.

18. Jardim FR, de Rossi FT, Nascimento MX, da Silva Barros RG, Borges PA, Prescilio IC and de Oliveira MR: Resveratrol and brain mitochondria: A review. Mol Neurobiol 55: 2085-2101, 2018.

19. Tauskela JS, Comas T, Hewitt K, Monette R, Paris J, Hogan M and Morley P: Cross-tolerance to otherwise lethal $\mathrm{N}$-methyl-D-aspartate and oxygen-glucose deprivation in preconditioned cortical cultures. Neuroscience 107: 571-584, 2001.

20. Ngan E, Stoletov K, Smith HW, Common J, Muller WJ, Lewis JD and Siegel PM: LPP is a Src substrate required for invadopodia formation and efficient breast cancer lung metastasis. Nat Commun 8: 15059, 2017.

21. Zheng Y, Gu S, Li X, Tan J, Liu S, Jiang Y, Zhang C, Gao L and Yang HT: Berbamine postconditioning protects the heart from ischemia/reperfusion injury through modulation of autophagy. Cell Death Dis 8: e2577, 2017.

22. Zinchuk V, Zinchuk O and Okada T: Quantitative colocalization analysis of multicolor confocal immunofluorescence microscopy images: Pushing pixels to explore biological phenomena. Acta Histochem Cytochem 40: 101-111, 2007.

23. Zhang X, Yan H, Yuan Y, Gao J, Shen Z, Cheng Y, Shen Y, Wang RR, Wang $\mathrm{X}, \mathrm{Hu} \mathrm{WW}$, et al: Cerebral ischemia-reperfusion-induced autophagy protects against neuronal injury by mitochondrial clearance. Autophagy 9: 1321-1333, 2013.

24. GBD 2016 Stroke Collaborators. Global, regional, and national burden of stroke, 1990-2016: A systematic analysis for the Global Burden of Disease Study 2016. Lancet Neurol 18: 439-458, 2019.

25. Schaller B and Graf R: Cerebral ischemia and reperfusion: The pathophysiologic concept as a basis for clinical therapy. J Cereb Blood Flow Metab 24: 351-371, 2004.

26. Moskowitz MA, Lo EH and Iadecola C: The science of stroke: Mechanisms in search of treatments. Neuron 67: 181-198, 2010.

27. Harikumar KB and Aggarwal BB: Resveratrol: A multitargeted agent for age-associated chronic diseases. Cell Cycle 7: 1020-1035, 2008

28. Petrovski G, Gurusamy N and Das DK: Resveratrol in cardiovascular health and disease. Ann N Y Acad Sci 1215: 22-33, 2011.

29. Tsai SK, Hung LM, Fu YT, Cheng H, Nien MW, Liu HY, Zhang FB and Huang SS: Resveratrol neuroprotective effects during focal cerebral ischemia injury via nitric oxide mechanism in rats. J Vasc Surg 46: 346-353, 2007.

30. Huang SS, Tsai MC, Chih CL, Hung LM and Tsai SK: Resveratrol reduction of infarct size in Long-Evans rats subjected to focal cerebral ischemia. Life Sci 69: 1057-1065, 2001.

31. Zhu J, Wang KZ and Chu CT: After the banquet: Mitochondrial biogenesis, mitophagy, and cell survival. Autophagy 9: 1663-1676, 2013

32. Decker RS and Wildenthal K: Lysosomal alterations in hypoxic and reoxygenated hearts. I. Ultrastructural and cytochemical changes. Am J Pathol 98: 425-444, 1980.

33. Zhang Z and Yu J: NR4A1 promotes cerebral ischemia reperfusion injury by repressing Mfn2-mediated mitophagy and inactivating the MAPK-ERK-CREB signaling pathway. Neurochem Res 43: 1963-1977, 2018.

34. Li F, Tan J, Zhou F, Hu Z and Yang B: Heat shock protein B8 (HSPB8) reduces oxygen-glucose deprivation/reperfusion injury via the induction of mitophagy. Cell Physiol Biochem 48: 1492-1504, 2018.

35. Ji W, Wei S, Hao P, Xing J, Yuan Q, Wang J, Xu F and Chen Y: Aldehyde dehydrogenase 2 has cardioprotective effects on myocardial ischaemia/reperfusion injury via suppressing mitophagy. Front Pharmacol 7: 101, 2016.

36. Andres AM, Hernandez G, Lee P, Huang C, Ratliff EP, Sin J, Thornton CA, Damasco MV and Gottlieb RA: Mitophagy is required for acute cardioprotection by simvastatin. Antioxid Redox Signal 21: 1960-1973, 2014. 
37. Guan R, Zou W, Dai X, Yu X, Liu H, Chen Q and Teng W: Mitophagy, a potential therapeutic target for stroke. J Biomed Sci 25: 87, 2018

38. Ashrafi G and Schwarz TL: The pathways of mitophagy for quality control and clearance of mitochondria. Cell Death Differ 20: 31-42, 2013.

39. Pineda-Ramírez N, Alquisiras-Burgos I, Ortiz-Plata A, Ruiz-Tachiquín ME, Espinoza-Rojo $M$ and Aguilera $P$ : Resveratrol activates neuronal autophagy through AMPK in the ischemic brain. Mol Neurobiol 57: 1055-1069, 2020.

40. Wang $\mathrm{H}$, Chen S, Zhang Y, Xu H and Sun H: Electroacupuncture ameliorates neuronal injury by Pink1/Parkin-mediated mitophagy clearance in cerebral ischemia-reperfusion. Nitric Oxide 91: 23-34, 2019.
41. He Q, Li Z, Meng C, Wu J, Zhao Y and Zhao J: Parkin-dependent mitophagy is required for the inhibition of ATF4 on NLRP3 inflammasome activation in cerebral ischemia-reperfusion injury in rats. Cells 8: 897, 2019.

(i) $($ ) This work is licensed under a Creative Commons Attribution-NonCommercial-NoDerivatives 4.0 International (CC BY-NC-ND 4.0) License. 